Bundesgesundheitsbl -

\section{Zusammenfassung}

In dem vorliegenden Beitrag wird zunächst über die Infektion der Schwangeren mit Toxoplasmen sowie über die Laboratoriumsdiagnostik bei der Mutter, dem Fötus und Neugeborenen berichtet.

Danach werden die Folgen für das Kind und die Chemotherapie dargestellt. Zudem wird eingehend die Vorbeugung und die Vorsorgeproblematik behandelt sowie auf die Meldepflicht eingegangen.

\section{Schlüsselwörter}

Toxoplasma-Infektion · Toxoplasmose · Schwangere · Schwangerenvorsorge . Chemotherapie · Laboratoiumsdiagnostik

Leitthema:Mutter-Kind-Übertragung von Infektionskrankheiten

K. Janitschke • Konsiliarlaboratorium Toxoplasma am Robert Koch-Institut, Berlin

\title{
Pränatale Übertragung der Toxoplasmen von der Mutter auf das Kind*
}

oxoplasmen sind einzellige Parasiten, die bei warmblütigen Tieren weit verbreitet sind und auf den Menschen übertragen werden können. Über neunzig Prozent der Infektionen des Menschen verlaufen ohne klinische Symptome. In den anderen Fällen kann es u.a. zu Abgeschlagenheit und leichtem Fieber sowie zu Lymphknotenschwellungen besonders im Halsbereich kommen. Schwere Verläufe mit häufig tödlichem Ausgang werden bei Immunsupprimierten, insbesondere bei AIDS- und Transplantationspatienten, beobachtet. Wegen der Häufigkeit der Fälle besitzt die Infektion ihre größte Bedeutung für den Schwangerschaftsverlauf und die Kindesentwicklung. Hierüber soll aus der Sicht des Konsiliarlaboratoriums und der langjährigen Geschäftsstelle der Beratenden Kommission „Toxoplasmose und Schwangerschaft" am Robert KochInstitut berichtet werden.

\section{Infektion der Mutter - Quellen, Häufigkeit}

Die Toxoplasmen-Infektion des Menschen kommt im wesentlichen durch Toxoplasma-Zysten und -Oozysten zustande. Im Fleisch, insbesondere von Schwein und Schaf, sind diese Zysten enthalten. Durch den Verzehr rohen oder ungenügend erhitzten Fleisches kann es zur Infektion kommen. Katzen, im wesentlichen nur erstmalig infizierte, können Oozysten mit dem Kot ausscheiden. Diese sind zunächst nicht sporuliert, d.h. nicht infektiös. Sie benötigen zur Sporulation wenigstens drei Tage Luft, Feuchtigkeit und Wärme. Daher ist davon auszugehen, daß der Kontakt mit der Katze selbst kein Infektionsrisiko darstellt. Dieses besteht jedoch durch Kontakt mit Erdboden, in dem die Oozysten mindestens ein Jahr überleben können, wenn es bei ungenügender Hygiene zur oralen Aufnahme kommt. Welcher Infektionsweg (ob Zyste oder Oozyste) heutzutage der wichtigere ist, kann nicht entschieden werden. Dabei ist zu berücksichtigen, daß Schweine in den sechziger Jahren eine Prävalenz der Infektion von ca. 90\% aufwiesen, die heute durch veränderte Haltungsbedingungen wesentlich gesunken ist. In Österreich ist die Anzahl der infizierten Schweine von $13,7 \%$ im Jahre 1982 auf 9,9\% im Jahre 1992 [1] gesunken. Damit könnte im Zusammenhang stehen, daß in den letzten zwei Jahrzehnten die Prävalenz bei Schwangeren von ca. 50\% auf unter $30 \%$ gefallen ist [2].

* Herrn Dir. und Prof. i.R. Dr. Hans Werner zum

75. Geburtstag zugeeignet

Dr. Klaus Janitschke

Robert Koch-Institut, Parasitologie und Mykologie, Postfach 650280, D-13302 Berlin 


\section{K. Janitschke}

\section{Prenatal transmission of toxoplasma from mother to child}

\section{Summary}

A report is given on the infection of pregnant women with Toxoplasma and on the laboratory diagnosis on mother, fetus and newborn. The next chapters deal with the consequences for the child and its chemotherapy. Prevention and problems of mother care are discussed in detail, as well as the infection as a notifiable one.

\section{Key words}

Toxoplasma-infection - Toxoplasmosis . Pregnancy - Mother care · Chemotherapy . Laboratory diagnosis
Eine sinkende Prävalenz bei Frauen im gebärfähigen Alter ist keineswegs ungeteilt positiv zu bewerten, denn damit steigt der Anteil der Frauen ohne Infektion und somit das Risiko einer Erstinfektion in der Schwangerschaft. Besteht dagegen bei Schwangeren eine ältere, vor der Gravidität entstandene und nun inaktive Infektion, so liegt auch eine Immunität und somit ein Schutz vor einem pränatalen Übergang des Erregers auf das ungeborene Kind vor.

\section{„Eine sinkende Prävalenz der Infekti- on bei Frauen im gebärfähigen Alter erhöht das Risiko einer Erstinfektion in der Schwangerschaft."}

Wie groß die Prävalenz von Schwangeren ist, ist somit Grundlage für die Abschätzung des Risikos von pränatalen Infektionen. Zwischen 34\% (BadenWürttemberg) und 54\% (Berlin) der Schwangeren sind infiziert $[3,4]$. Worauf diese räumlich differierenden Unterschiede beruhen, ist nicht geklärt. Die ehemalige Annahme, daß der Unterschied im hohen Ausländeranteil an der Bevölkerung Berlins beruht, scheint spekulativ zu sein. Immerhin ist festzuhalten, daß bei uns die Hälfte bis zu Zweidrittel der Schwangeren keine Immunität besitzt und somit das Risiko einer Erstinfektion während der Gravidität besteht. Die Infektion verläuft auch bei Schwangeren in über neunzig Prozent der Fälle ohne Symptome, ist also als Erkrankung nicht erkennbar. Das steht im Gegensatz zu der Festlegung in der Mutterschaftsvorsorge, wobei es heißt, eine serologische Untersuchung kann vorgenommen werden bei „begründetem Verdacht auf Toxoplasmose“.

Der Ausschuß Mutterschaftsrichtlinien der KBV legt dies jedenfalls klinisch aus, nur bei wenigen Frauen kommt es jedoch zur Erkrankung der Toxoplasmose. Ein Verdacht muß u.E. auch epidemiologisch begründet werden, da sehr viele Frauen rohes oder ungenügend erhitztes Fleisch essen und Kontakt mit Erdboden haben. Das wichtigste Argument für eine weitergehende Auslegung der Mutterschaftsrichtlinien ist, daß die meisten Schwangeren, die den Parasiten auf ihr Kind übertragen, keinerlei Krankheitszeichen aufwiesen. Es wird daher die Erweiterung der Mutterschaftsvorsorge auf alle Schwangeren, nicht nur auf die klinisch verdächtigen, gefordert. Das setzt eine qualitativ hochwertige Serodiagnostik voraus.

\section{Serodiagnostik bei Frauen}

Durch die Untersuchung von Serum auf spezifische Antikörper kann festgestellt werden, ob eine Infektion vorliegt oder nicht, und ob es sich um eine aktive oder inaktive handelt. Jahrzehntelang wurde dazu um Methoden und Interpretationen von Ergebnissen gestritten. Diese Diskussionen sind noch nicht ganz verstummt, belasten aber weiterhin das Vertrauen von einem Teil maßgeblicher Kreise in die Sicherheit der Serodiagnostik bei Schwangeren. Auch die INSTAND-Ringversuche zur externen Qualitätssicherung der Toxoplasmose-Serologie zeigen, daß es immer noch einzelne Laboratorien gibt, die Qualitätsanforderungen nicht erfüllen.

\section{"Die Toxoplasma-Infektion verläuft bei Schwangeren in über neunzig Prozent der Fälle symptomlos und ist daher als Erkrankung nicht erkennbar."}

Es müssen daher strikte Maßstäbe angelegt werden. Das vormalige Fachgebiet Klinische Parasitologie im RKI hat hierzu einen Schwerpunkt seiner Arbeit gesehen. Es wurde eine Methode zur stufenweisen Diagnostik entwickelt, die die Beratende Kommission „Toxoplasmose und Schwangerschaft" in ihre Empfehlungen zur Laboratoriumsdiagnostik übernommen hat und die auch in die GOÄ der KBV einflossen. Die Vorgehensweise wurde von der Kommission kürzlich an die aktuellen Verhältnisse angepaßt $[5,6]$. Bei sorgfältiger und kritischer Vornahme der serologischen Teste dürften nahezu alle Fälle dahingehend abzuklären sein, ob eine Relevanz für die Schwangerschaft besteht oder nicht. Zur weiteren Sicherung der Qualität der Laboratoriumsuntersuchungen hat die Kommission die Einführung der Zulas- 


\section{Leitthema:Mutter-Kind-Übertragung von Infektionskrankheiten}

sungs- und Chargenprüfpflicht für Toxoplasmose-Diagnostik durch das PEI erbeten, die am 6. Juli 1993 festgeschrieben wurde. Eine Zulassungspflicht für Toxoplasmose-Diagnostika besteht auch nach der In-vitro-Diagnostika Richtlinie der EU, die am 1.7.1998 erlassen wurde. Als Voraussetzung für die Erweiterung der Mutterschaftsvorsorge fordert die Kommission auch die erfolgreiche Pflichtteilnahme an INSTAND-Ringversuchen (Parasitenimmunologie I) zur externen Qualitätssicherung. Schließlich hat die Kommission eine Reihe von Beratungslaboratorien und ein Konsiliarlaboratorium benannt, die bei der Klärung von Problemfällen Hilfestellung leisten können [7].

Sehr hilfreich für die Klassifizierung von Infektionen ist auch eine $\mathrm{Pu}$ blikation der EU-Arbeitsgruppe „Konnatale Toxoplasmose" [8]. Wir sehen heutzutage keinen stichhaltigen Grund mehr, die Validität der Serodiagnostik der Toxoplasma-Infektion in Frage zu stellen, sofern die geforderten Qualitätsmaßstäbe angelegt werden.

\section{Pränatale Übertragung}

Wurde durch serodiagnostische Untersuchungen festgestellt, daß sich die Schwangere erstmalig mit Toxoplasmen infiziert hat, so besteht das Risiko der Übertragung des Parasiten auf das ungeborene Kind. Toxoplasmen können zu jedem Zeitpunkt der Gravidität übertragen werden und gehen in der ersten Hälfte der Schwangerschaft in 4 bis $15 \%$ und in der zweiten Hälfte in ca. $60 \%$ der Fälle auf den Fetus über. Kommt es dadurch nicht zum Abort, so kann es zu schweren Schädigungen wie Enzephalitis, Hydrozephalus, intrazerebralen Verkalkungen oder Retinochorioiditis kommen. Je älter die Schwangerschaft zum Zeitpunkt der Erstinfektion ist, um so mehr nimmt der Grad der Schädigungen des Kindes ab und das Risiko der Übertragung zu.

Für die Erkennung einer pränatalen Infektion hat die Kommission am RKI ebenfalls Empfehlungen erarbeitet. Klinisch ist eine Schädigung des Fetus ab der 22. Schwangerschaftswoche durch Ultraschall feststellbar. Labordiagno-

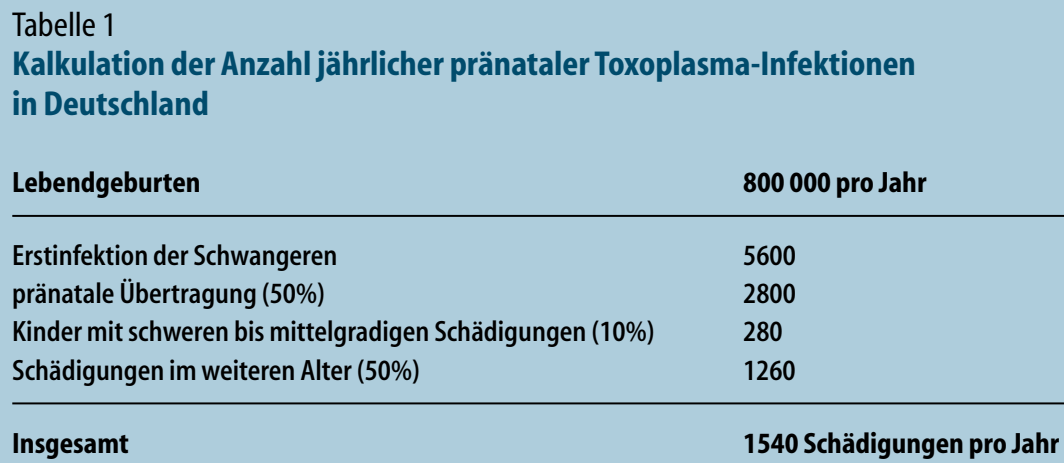

Insgesamt

1540 Schädigungen pro Jahr

stisch kann Fruchtwasser mittels PCR auf Toxoplasma-DNS untersucht werden. Nur in Einzelfällen kann heute die zusätzliche Untersuchung von fötalem Blut empfohlen werden. Die Indikation zur PCR-Untersuchung muß sehr kritisch abgewogen werden. Dabei ist vor allem abzuklären, ob bei der Schwangeren eine Erstinfektion gesichert, wahrscheinlich oder möglich ist [8]. Weder ein positives noch ein negatives PCR-Ergebnis für sich allein läßt den Schluß zu, ob eine pränatale Infektion vorliegt oder nicht. Dazu bedarf es der Zusammenführung aller vorliegenden klinischen und labordiagnostischen Daten. Erst nach solcher kritischen Befundung des jeweiligen Einzelfalles kann das weitere Vorgehen hinsichtlich einer ggf. notwendigen Chemotherapie festgelegt werden.

\section{„Je älter die Schwangerschaft zum} Zeitpunkt der Toxoplasmen-Infektion ist, desto geringer ist der Grad der Schädigung des Kindes, aber desto höher wird das Risiko der Übertragung."

Dies hat in einem eingehenden Gespräch mit einem erfahrenen Arzt zu geschehen, was besonders im Hinblick auf einen von der Schwangeren gewünschten Abort notwendig ist. Die Erfahrung zeigt, daß bei dieser Vorgehensweise ein wesentlicher Teil der Schwangeren nicht auf einen Abort besteht. Die in diesem Heft (Seite 610) genannte Liste der Beratungsstellen enthält neben denen zur Diagnostik auch solche, die bei klinischen und diagnostischen Problemen beratend helfen können [7].

Bei der Geburt können nur etwa $10 \%$ der infizierten Kinder klinisch erkannt werden. Bei den übrigen kann eine Infektion zunächst nur serologisch festgestellt werden, wozu die Beratende Kommission ebenfalls Empfehlungen erarbeitet hat (siehe auch Beitrag Seite 606 dieses Heftes) $[5,6]$. Die Hälfte der zunächst klinisch stummen Fälle kann jedoch im Laufe der ersten ein bis drei Lebensjahrzehnte Erscheinungen wie Retinochorioiditis und mentale Retardierung entwickeln.

\section{Häufigkeit pränataler Infektionen}

Die Frage der Häufigkeit pränataler Toxoplasma-Infektionen in Deutschland wird seit langem kontrovers diskutiert. Die Beratende Kommission am RKI „Toxoplasmose und Schwangerschaft" hat aufgrund eigener Daten der Mitglieder sowie der Auswertung der Weltliteratur, besonders der Mitteleuropas, eine Häufigkeit von sieben pränatalen Toxoplasma-Infektionen pro 1000 Lebendgeburten errechnet [9] - daraus ergibt sich für Deutschland pro Jahr eine in Tabelle 1 dargestellte Kalkulation. Dieser Kalkulation steht jene des Ausschusses Muttterschaftsrichtlinien der KBV gegenüber. Sie geht von jährlich maximal 250 Fällen aus, wobei nur 100 Fälle eher realistisch seien [10].

Der o.g. Ausschuß argumentiert vor allem mit den Zahlen der gemeldeten Fälle pränataler Toxoplasma-Infektionen nach dem Bundesseuchengesetz. Diese 
lagen in den Jahren 1986 bis 1990 bei jährlich 17 bis 15, dies ist ein erheblicher Unterschied zu der erwarteten Fallzahl. Tauchmann hat dies in einer Studie in Berlin näher untersucht [11]. Die Autorin kommt zu dem Schluß, daß eine Reihe von Meldungen nicht erfolgte und ein Teil der gemeldeten Fälle einer kritischen Prüfung nicht standhielt.

\section{"Die Kommission Toxoplasmose und Schwangerschaft am RKI geht in Deutschland von jährlich rund 1500 Schädigungen aufgrund pränataler Toxoplasma-Infektionen aus."}

Das liegt nicht nur an der Meldemüdigkeit, sondern auch am Fehlen einer klaren Definition für eine pränatale Toxoplasmose. Durch die Falldefinition der o.g. EU-Arbeitsgruppe [8] ist aber nunmehr eine bessere Basis für die Meldungen gegeben.

\section{Chemotherapie bei Mutter und Kind}

Pränatale Toxoplasma-Infektionen können durch eine Chemotherapie verhindert werden, was z.B. bei einer pränatalen Rötelninfektion nicht möglich ist. Die Kommission hat auch hierzu Empfehlungen erarbeitet [5]. Danach sollte bis einschließlich der 15. Schwangerschaftswoche Spiramycin gegeben werden. Zur Vermeidung möglicher Risiken kann erst ab der 16. Woche über vier Wochen die Dreifachkombination Sulfadiazin, Pyrimethamin und Folinsäure unter wöchentlicher Kontrolle des Blutbildes dargereicht werden. Bessere Erfolge durch eine längere Therapiedauer können nicht belegt werden. Nur im Falle der Infektion des Fetus sollte unter Wechsel der Einfach- mit der Dreifachmedikation bis zum Ende der Schwangerschaft behandelt werden. Kontrovers wurde auch hier mit dem Ausschuß Mutterschaftsrichtlinien der Prozentsatz eines Therapieerfolges diskutiert. Er geht von einer Rate von 50 bis $60 \%$ aus.

Daß eine Chemotherapie nicht alle Fälle von pränatalen Infektionen verhindern kann, muß hingenommen werden. Je früher eine Erstinfektion bei einer
Schwangeren erkannt wird, um so erfolgreicher dürfte eine Chemotherapie sein. Das Bekämpfungsprogramm in Österreich zeigte, daß im Laufe seines 25jährigen Bestehens bei keiner Frau, die sich regelrecht der Diagnostik und Therapie unterzogen hatte, ein pränatal infiziertes Kind festgestellt werden konnte [12].

Diese Tatsache spricht eindeutig für ein Vorbeugeprogramm. Bei uns könnten unter Annahme eines Therapieerfolges von $80 \%$ mindestens 1232 Kinder bzw. spätere Erwachsene vor den Folgen einer pränatalen Infektion geschützt werden. Kam es jedoch durch das Versäumnis der regelrechten Untersuchung der Schwangeren oder durch andere Fehler zu einer pränatalen Infektion, so kann auch in diesen Fällen postnatal chemotherapeutisch behandelt werden. Bewährt hat sich dazu die zunächst vierwöchige Anwendung der Dreifachkombination Sulfadiazin, Pyrimethamin und Folinsäure, anschließend kann ggf. mit Spiramycin therapiert werden.

\section{Vorbeugung}

Maßnahmen zur Vorbeugung einer Erstinfektion von Schwangeren sind ein unverzichtbarer Bestandteil der gesundheitlichen Aufklärung in der Schwangerenvorsorge. Die Schwangere sollte kein rohes oder ungenügend erhitztes Fleisch essen und sich nach dem Zubereiten von rohem Fleisch in der Küche gründlich die Hände waschen, dies gilt auch für das Arbeiten am oder im Erdboden.

Katzen können im Hause gelassen werden, sollten jedoch nur mit gekochtem Futter oder Pellets gefüttert werden. Ggf. vorhandene Katzentoiletten sind täglich durch andere Personen mit heißem Wasser zu reinigen. Frauen sollten sich möglichst kurz vor oder am Beginn einer Schwangerschaft ggf. auch während dieser auf Toxoplasma-Antikörper prüfen lassen. Darin sehen wir die wichtigste Vorbeugemaßnahme und fordern daher die Erweiterung der Mutterschaftsvorsorge auf alle Schwangeren. Hinweise auf Vorsorgemaßnahmen sind im Merkblatt für Ärzte „Toxoplasmose und Schwangerschaft" des RKI und des BgVV (siehe Beitrag Seite 606 dieses Heftes) [5] sowie in der Broschüre „Schwangerschaft“ der
BZgA und in Mitteilungen von Firmen der Diagnostikindustrie. In Österreich befindet sich ein entsprechender Beilagezettel im Mutterpaß.

\section{„Die Kombination von Aufklärung und Screening ist als der effektivste Weg zur Senkung der Zahl präna- taler Infektionen anzusehen."}

Immer wieder wird die Frage diskutiert, ob an Stelle eines umfassenden serologischen Screenings von Schwangeren eine intensive Aufklärung ausreichen würde. Ein Effekt von Aufklärungsmaßnahmen ist sicher vorhanden, es kann jedoch nicht erwartet werden, daß allein damit die Zahl pränataler Infektionen deutlich gesenkt werden könnte. Die Kombination von Aufklärung und Screening ist als der effektivste Weg anzusehen.

\section{Bekämpfungsprogramme}

In einer Reihe von Ländern wird wiederholt die Frage der Einführung eines landesweiten Screenings der Schwangeren oder Neugeborenen diskutiert. Befürworter und Gegner bringen ihre Argumente vor, so daß bis auf wenige Ausnahmen keine Bekämpfungsprogramme etabliert werden konnten. Als erstes Land führte Österreich im Jahre 1975 das allgemeine Screening der Schwangeren ein. Lag in den 5oer und 6oer Jahren die Rate der pränatal infizierten Kinder bei 50 bis 70 auf 1000 Geburten, so beträgt sie heute ein bis zwei auf 10 ooo Kinder. Die wenigen jetzt auftretenden Fälle pränataler Toxoplasmose sind, soweit bekannt, durchweg auf Fehler in der Vorsorge und Therapie zurückzuführen [11]. Der Rückgang der Prävalenz bei den Schwangeren in den letzten Jahren wird nicht als Argument gegen die Weiterführung des Screenings gesehen, da durch die Abnahme der Zahl immuner Schwangerer sich das Risiko der Erstinfektion während der Schwangerschaft vergrößert. Frankreich führte im Jahre 1978 das allgemeine Screening der Heiratswilligen bzw. Schwangeren ein. Daten über die Effektivität des Programms wurden jedoch nicht erhoben. 
Die Einführung des Screenings in der DDR im Jahre 1985 war damals politisch gewollt. Es sollte die Säuglingssterblichkeit deutlich gesenkt werden, und als Ursache dafür galt auch die Toxoplasmose. Unter den Bedingungen eines zentral organisierten Staates wurde ein wohldurchdachtes System der Schwangeren- und Kindervorsorge aufgebaut. Bis 1989 verblieb jedoch nur eine relative kurze Zeit, so daß sich erst ca. die Hälfte der Schwangeren dem Screening unterzog. Die Erfahrungen aus diesen Programmen sind in die Arbeiten der BGA/RKI-Kommission „Toxoplasmose und Schwangerschaft" eingeflossen.

In Dänemark gibt es seit Juni 1998 ein allgemeines Screening der Neugeborenen auf pränatale Toxoplasma-Infektionen.

\section{Mutterschafts-Richtlinien}

In der alten und neuen Bundesrepublik Deutschland gelten die MutterschaftsRichtlinien. Sie besagen, daß wenn ein Arzt bei einer Schwangeren einen begründeten Verdacht auf Toxoplasmose hat, so kann eine Untersuchung auf Antikörper vorgenommen und nach den Richtlinien abgerechnet werden. Die KBV legt den begründeten Verdacht klinisch aus, jedoch verlaufen über $90 \%$ der Erstinfektionen klinisch unauffällig. Dennoch kommt es in der Hälfte der Fälle zu einer pränatalen Übertragung. Das Risiko einer Erstinfektion geht vom Rohfleischverzehr und vom Kontakt mit Erdboden aus. Daher ist nach unserer Ansicht die Formulierung „begründeter Verdacht" auch epidemiologisch auszulegen. Die Kommission fordert seit 1991 die Erweiterung der MutterschaftsRichtlinien. Dem steht die Auffassung des zuständigen Ausschusses der KBV entgegen, die ihre Ablehnung unserer Vorschläge u.a. mit Zahlen über die Prävalenz der Infektion begründet, die auf ungenügenden Literaturstudien basiert; sie verstieg sich bis zu der Äußerung, daß ein Toxoplasmose-Screening unethisch sei, weil der Schaden größer als der Nutzen sei [12]. Die Kosten-Nutzen-Kalkulation unserer Kommission ergab einen 2,6fach höheren Nutzen durch Screening und ggf. Behandlung der Schwangeren.
Um eine sichere Datenbasis über Prävalenz zu schaffen, hat die Kommission eine Studie an Neugeborenen vorgeschlagen. Durch widerstreitende Diskussionen konnte das Projekt nicht in Angriff genommen werden. Ein solches Programm besäße derzeit keine gesundheitspolitische Priorität, heißt es.

\section{Aktuelle Situation}

Die Forderung der Kommission „Toxoplasmose und Schwangerschaft" hinsichtlich der Ausdehnung der Mutterschafts-Richtlinien auf alle Schwangeren wird weiter erhoben. Die KBV antwortete darauf mit ihrer Entscheidung, Toxoplasmose-Untersuchungen, sofern sie nicht unter die begrenzten Mutterschafts-Richtlinien fallen, in die IGELListe aufzunehmen. Danach sollen die Schwangeren die Untersuchungen als individuelle gesundheitliche Eigenleistung selbst bezahlen.

Die Konnatale Toxoplasmose ist als Erkrankung und Tod in der Liste der meldepflichtigen Krankheiten im Bundesseuchengesetz aufgeführt. Im Entwurf des Infektionsschutzgesetzes ist sie ebenfalls aufgenommen mit der Begründung, $\mathrm{da} ß$ die Toxoplasmose in der Zoonosenrichtlinie der EU enthalten ist. Die Kommission Toxoplasmose und Schwangerschaft hält es für notwendig, daß die Infektion in das Gesetz übernommen wird. Man erwartet, daß damit verläßliche Daten über die Häufigkeit der pränatalen Toxoplasmose gewonnen werden können. Grundlage für die Meldung ist eine klare Falldefinition, die vorgeschlagen wurde. Diese stützt sich im einzelnen, zu entscheidenden Fall auf die Klassifikation und Falldefinition der Arbeitsgruppe Konnatale Toxoplasmose der EU [8]. Darin wird aufgeführt, bei welchen klinischen und serologischen Fakten eine Infektion bei einer Schwangeren bzw. bei einem Neugeborenen gesichert, wahrscheinlich bzw. möglich ist oder als unwahrscheinlich gilt bzw. ausgeschlossen ist. Es wird vorgeschlagen, daß die nach dem Infektionsschutzgesetz eingehenden Meldungen von Fällen pränataler Toxoplasmose durch erfahrene Experten hinsichtlich der Meldekriterien beurteilt werden. Dadurch wäre es möglich, gesi- cherte Daten über die Häufigkeit von Fällen zu erhalten, um damit auch die Wirkung gesundheitlicher Vorsorgemaßnahmen beurteilen zu können.

\section{Weitere Hinweise}

In diesem Heft finden Sie zum Thema Toxoplasmose weitere Beiträge in der Rubrik Bekanntmachungen (Seite 6o6ff.).

\section{Literatur}

1. Edelhofer R (1994) Prevalance of antibodies against Toxoplasma gondii in pigs in Austria - an evaluation of data from 1982 and 1992. Parasit Res 80:642-644

2. Aspöck H, Pollak A (1992) Prevention of prenatal toxoplasmosis by serological screening of pregnant women in Austria. Scand J Inf Dis [Suppl] 84:32-38

3. Hlobil H, Gültig K, Naser K, Nötzel K (1992) Konnatale Toxoplasma-Infektionen in BadenWürttemberg. Klin Lab 38:67a-68b

4. Janitschke K, Busch W, Kellershofen C (1988) Untersuchungen zur Anwendbarkeit der direkten Agglutination zur ToxoplasmoseÜberwachung im Rahmen der Mutterschaftsvorsorge. Imm Inf 16:189-191

5. Robert Koch-Institut und Bundesinstitut für gesundheitlichen Verbraucherschutz und Veterinärmedizin (1999) Toxoplasmose bei Mutter und Kind. Erkennung, Behandlung und Verhütung. Merkblatt Bundesgesundhbl 42:606-609

6. Janitschke K, Hlobil H (1998) Aktuelle Empfehlungen zur Vorgehensweise bei der Untersuchung auf Toxoplasma-Antikörper bei Schwangeren, Neugeborenen und Kleinkindern. J Lab Med 22:495-498

7. Robert Koch-Institut (1999) Beratungsstellen für die Laboratoriumsdiagnostik sowie Klinik und Therapie der Toxoplasma-Infektion bei der Schwangeren- und der Kindervorsorge. Bundesgesundhbl 42:610-611

8. Robert Koch-Institut (1997) Klassifizierung und Falldefinition pränataler ToxoplasmaInfektionen. European Research Network on Congenital Toxoplasmosis. Bundesgesundhbl 40:507-508

9. Janitschke K (1996) Toxoplasmose-Vorsorge bei Schwangeren und Neugeborenen in Deutschland. Mitt Österr Ges Tropenmed Parasit 18:19-24

10. Abholz HH (1993) Toxoplasmosescreening in der Schwangerschaft: mehr Schaden als Nutzen. Gesundh Wes 55:410-413

11. Tauchmann C (1986) Erfahrungen mit der Meldepflicht von Connataler Toxoplasmose Eine kritische Analyse über die von 1980-1984 nach dem Bundesseuchengesetz gemeldeten Fälle am Beispiel von Berlin West. Med Diss FU Berlin

12. Aspöck H (1996) Österreichs Beitrag zur Toxoplasmose-Forschung und 20 Jahre Toxoplasmose-Überwachung in Österreich. Mitt Österr Ges Tropenmed Parasit 18:1-18 